\title{
Multiscale origin of the magnetocaloric effect in Ni-Mn-Ga shape-memory alloys
}

\author{
Jordi Marcos, Lluís Mañosa, and Antoni Planes \\ Departament d'Estructura i Constituents de la Matèria, Facultat de Física, Universitat de Barcelona, Diagonal, 647, \\ E-08028 Barcelona, Catalonia, Spain
}

Fèlix Casanova, Xavier Batlle, and Amílcar Labarta

Departament de Física Fonamental, Facultat de Física, Universitat de Barcelona, Diagonal, 647, E-08028 Barcelona, Catalonia, Spain

(Received 3 February 2003; revised manuscript received 30 May 2003; published 3 September 2003)

\begin{abstract}
We have analyzed magnetization measurements in a series of composition-related Ni-Mn-Ga shape-memory alloys. It is shown that the magnetocaloric effect in the vicinity of the martensitic transition mainly originates from two different contributions: (i) magnetostructural coupling on the mesoscopic scale between the magnetic moments and the martensitic variants, which is also responsible for the magnetic shape-memory effect and (ii) the microscopic spin-phonon coupling which gives rise to the shift of the transition temperature with the applied magnetic field. The relative importance of these two contributions has been shown to vary with composition, which is suitably expressed through the average number of valence electrons per atom $e / a$. In alloys with a large difference between the Curie and martensitic transition temperatures $(e / a \simeq 7.5)$, mesoscopic coupling is dominant and a negative giant magnetocaloric effect (increase of temperature by adiabatic demagnetization) is induced at moderate applied fields. In contrast, in alloys when these temperatures are very close to one another $(e / a \simeq 7.7)$, the microscopic coupling is the most relevant contribution and gives rise to a positive giant effect.
\end{abstract}

DOI: 10.1103/PhysRevB.68.094401

PACS number(s): 75.80.+q, 81.30.Kf, 75.30.Sg

\section{INTRODUCTION}

$\mathrm{Ni}-\mathrm{Mn}-\mathrm{Ga}$ is a ferromagnetic alloy with a Curie temperature slightly above room temperature (weakly dependent on composition) that undergoes a martensitic transition at a temperature $T_{M}$ which is strongly sensitive to composition. ${ }^{1}$ This structural transition is responsible for the shape-memory properties $^{2}$ displayed by these materials including the magnetic shape-memory effect. ${ }^{3,4}$ Such a magneto-structural effect refers to the possibility of inducing giant deformations (in some cases more than 5\%) by the application of a moderate magnetic field (less than $10 \mathrm{kOe}$ ) in the martensitic phase. Interestingly, this exotic property has been observed not only in ferromagnetic, but also in antiferromagnetic materials. ${ }^{5}$

It has recently been reported that, in the vicinity of the martensitic transition, the application of a magnetic field induces a large change of entropy. ${ }^{6,7}$ Such a magnetocaloric effect ${ }^{8}$ is of great technological interest for cryogenic applications and has been intensively studied in recent years, especially after the discovery of giant magnetocaloric materials such as $\mathrm{Gd}_{5}\left(\mathrm{Si}_{x} \mathrm{Ge}_{1-x}\right)_{4}$ (Ref. 9) and MnAs-based compounds. ${ }^{10,11}$ In nearly stoichiometric $\mathrm{Ni}_{2} \mathrm{MnGa}$ alloys, the magnetocaloric effect has been shown ${ }^{12}$ to be strongly dependent on the changes of the domain mesostructure of the system induced by the application of a magnetic field. These changes are known to be controlled by the cross-correlation between structural and magnetic domains and is also responsible for shape-memory properties. This effect is, however, expected to lose importance as the martensitic transition approaches the Curie temperature. With this idea in mind, in the present paper we have analyzed magnetization data recently reported in a series of composition-related Ni-Mn-Ga alloys with $T_{M} \lesssim T_{c}$ that have compositions with an average number of valence electrons per atom $e / a \lesssim 7.7 .^{13}$ The evolution of the magnetostructural properties as $T_{M}$ approaches $T_{c}$ shows the multiscale origin of the magnetocaloric effect. Results are expected to be of interest in order to select the appropriate materials with optimal magnetocaloric performances for technological applications.

\section{MODELING AND RESULTS}

Magnetization measurements at different temperatures through the martensitic transition have recently been reported $^{6,7,12,14}$ for five alloys with different compositions. The composition and characteristic temperatures of these alloys are listed in Table I. From the reported magnetization

TABLE I. Atomic composition and transition temperatures of the alloys analyzed.

\begin{tabular}{|c|c|c|c|c|c|c|c|}
\hline $\begin{array}{l}\text { Alloy } \\
\text { No. }{ }^{a}\end{array}$ & Ref. & at. $\% \mathrm{Ni}$ & at. $\% \mathrm{Mn}$ & at. $\% \mathrm{Ga}$ & $e / a$ & $T_{M}(\mathrm{~K})$ & $T_{c}(\mathrm{~K})$ \\
\hline 1 & 12 & 49.5 & 25.4 & 25.1 & 7.48 & 180 & 381 \\
\hline 2 & 6 & 51.5 & 22.7 & 25.8 & 7.51 & 192 & 351 \\
\hline 3 & 7 & 52.6 & 23.1 & 24.3 & 7.61 & 294 & 345 \\
\hline 4 & 14 & 55.1 & 19.2 & 25.6 & 7.63 & 309 & 335 \\
\hline 5 & 14 & 56.2 & 18.2 & 25.6 & 7.66 & 361 & 361 \\
\hline
\end{tabular}

The lattice parameters determined for alloy 1 are $a=b=c$ $=5.817 \AA$ for the parent phase $(T=295 \mathrm{~K})$ and $a=b=5.92 \AA$, $c=5.57 \AA$ for the martensitic phase $(T=4.2 \mathrm{~K})$, while those corresponding to alloy 3 (taken from Ref. 7) are $a=b=c=5.828 \AA$ for the parent phase $(T=350 \mathrm{~K})$ and $a=b=5.923 \AA, c$ $=5.556 \AA$ for the martensitic phase $(T=250 \mathrm{~K})$. 


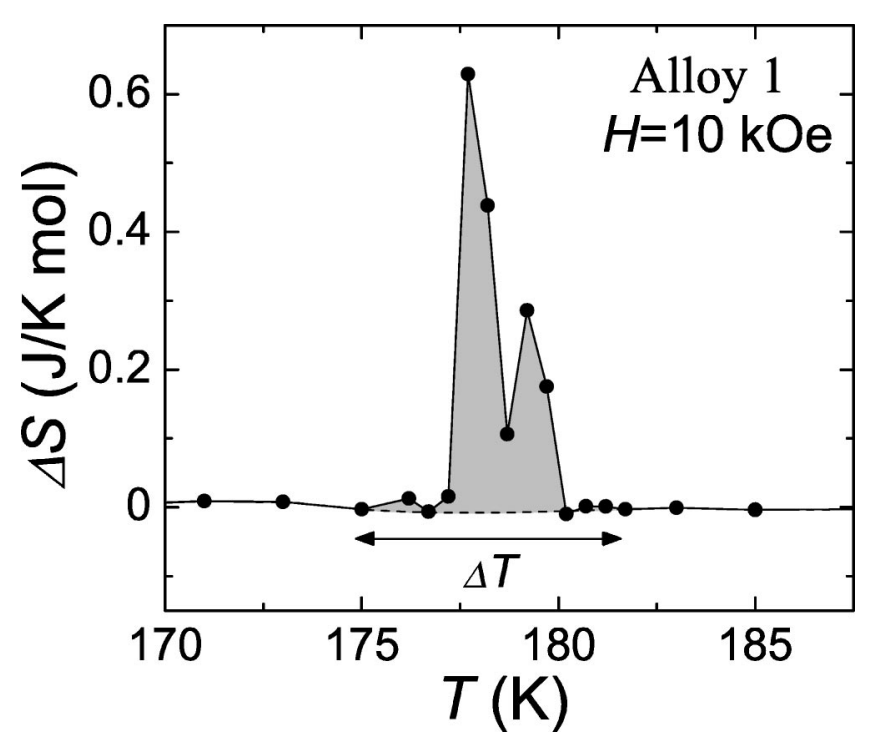

FIG. 1. Example of the entropy change obtained from magnetization curves using the Maxwell relation, which illustrates the procedure to compute the average values of the field-induced entropy change over the transformation region $\Delta T$.

data, the field-induced entropy change (as the field is increased from 0 to $H$ ) can be obtained as

$$
\Delta S(T, H)=\int_{0}^{H}\left(\frac{\partial M}{\partial T}\right)_{H} d H .
$$

In systems transforming martensitically, the temperature dependence of $\Delta S(T, H)$ for a given field is strongly influenced by discontinuities in the transformation path which are caused by (unavoidable) defects, composition inhomogeneities, etc. This discontinuous behavior gives rise to a $\Delta S(T, H)$ that shows peaks over a temperature range $\Delta T(H) .{ }^{12}$ This behavior is illustrated in Fig. 1. In order to compare $\Delta S(T, H)$ for different samples it is then convenient to calculate, for each field, the average of the field-induced entropy change over $\Delta T(H)$ as

$$
\langle\Delta S(H)\rangle=\frac{1}{\Delta T(H)} \int_{\Delta T} \Delta S(T, H) d T .
$$

This integral is performed numerically by taking a suitable base line which enables elimination of the contribution to $\langle\Delta S(H)\rangle$ arising from any possible temperature variation of the magnetization outside the transformation region. The method of integration is also illustrated in Fig. 1. The obtained values of $\langle\Delta S(H)\rangle$ as a function of the applied field for the five analyzed alloys are shown in Fig. 2. This entropy change first increases with $H$, then reaches a positive maximum, and linearly decreases for high fields. Except for alloys 1 and 2, the initial increase of $\langle\Delta S(H)\rangle$ is very weak and is not even observed for sample 5. It is worth noting that the maximum value of $\Delta S(T, H), \Delta S_{\max }(H)$, follows the same qualitative behavior as $\langle\Delta S(H)\rangle$.

In order to gain a deeper understanding of the results presented in Fig. 2, it is interesting to analyze the behavior in detail of the magnetization as a function of temperature, at

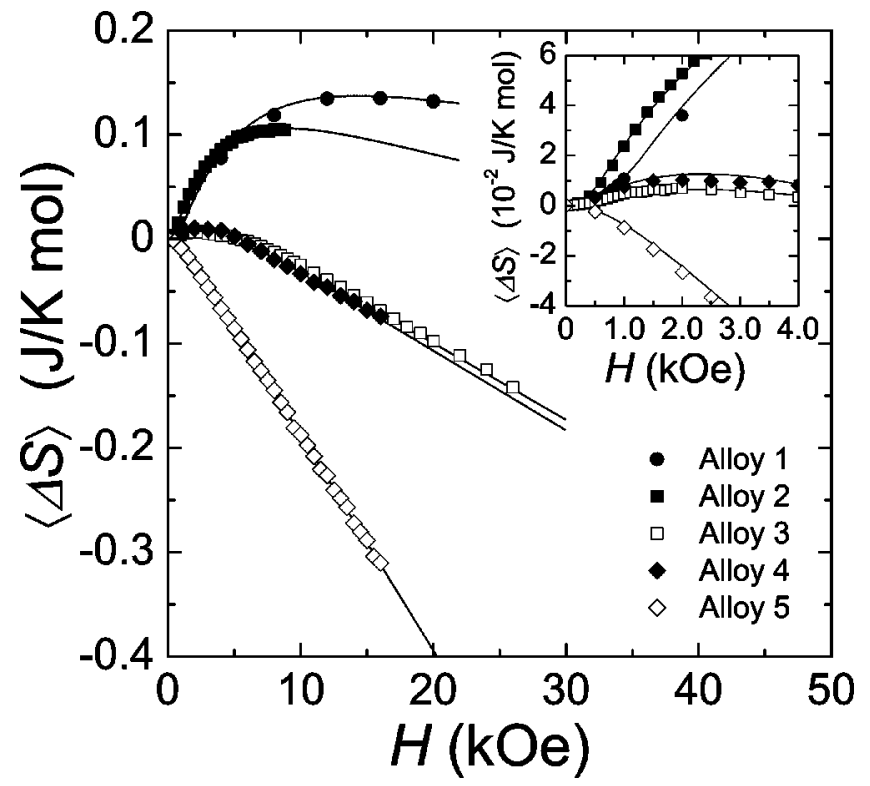

FIG. 2. Average field-induced entropy change $\langle\Delta S\rangle$ as a function of $H$ for all the different analyzed alloys. The continuous lines are the fits of Eqs. (10) $\left(H<H^{*}\right)$ and (11) $\left(H>H^{*}\right)$ to the experimental data. For these fits, the values of $H^{*}, H_{c}, \mu$, and $\Delta M_{\text {sat }}$ are obtained from the previous fit of the $\Delta M(H)$ curves [Eq. (6)]. $\Delta T$ is the only free parameter (see text for more details). The inset is an expanded view of the low-field region.

given applied fields, in the vicinity of the martensitic transformation. The most relevant feature is the abrupt change, $\Delta M$ (magnetization difference between martensite and parent phases), which occurs at the martensitic transition. In Fig. 3, we have plotted $\Delta M$ vs $H$ for all the analyzed samples. It is observed that $\Delta M$ significantly depends on the

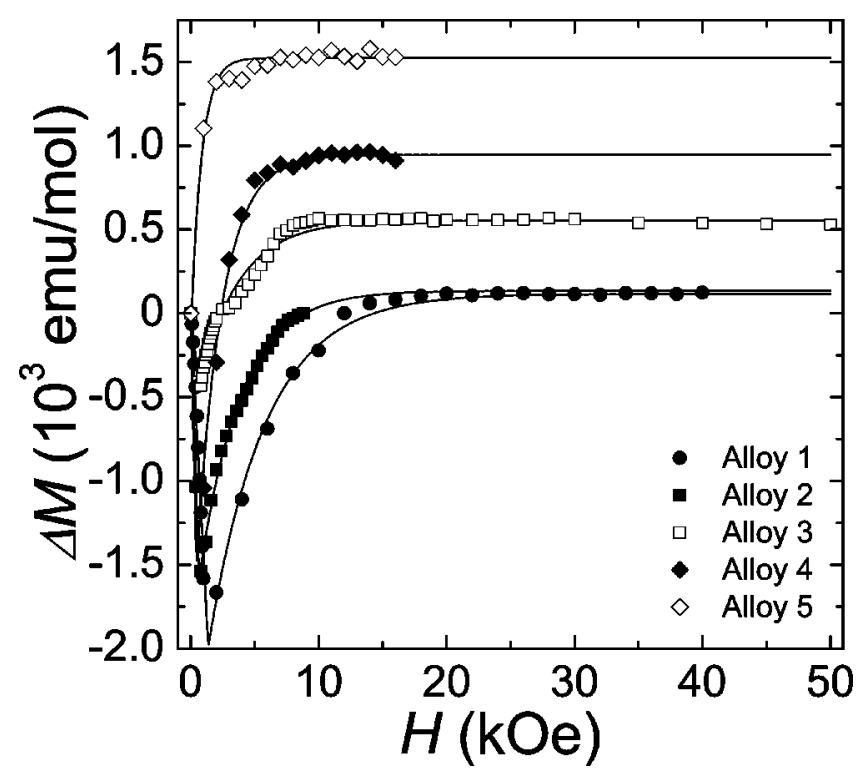

FIG. 3. Magnetization difference $\Delta M$ between martensite and parent phases as a function of $H$ for all the analyzed alloys. The continuous lines correspond to fits of Eq. (6) to the experimental data. The values of the fitted parameters $\left(H^{*}, H_{c}, \mu\right.$, and $\left.\Delta M_{\text {sat }}\right)$ are listed in Table II. 
TABLE II. Magnetic and thermodynamic quantities for the alloys analyzed.

\begin{tabular}{cccccccc}
\hline \hline Alloy No. & $\Delta S_{t}$ & $|\Delta T|^{\mathrm{a}}$ & $\frac{1}{\mu}$ & $\Delta M_{\text {sat }}$ & $H^{* \mathrm{~b}}$ & $H_{c}{ }^{\mathrm{c}}$ & $d T_{M} / d H$ \\
& $(\mathrm{~J} / \mathrm{K} \mathrm{mol})$ & $(\mathrm{K})$ & $(\mathrm{emu} / \mathrm{mol})$ & $(\mathrm{Oe})$ & $(\mathrm{Oe})$ & $(\mathrm{mK} / \mathrm{kOe})$ \\
\hline 1 & -0.550 & 6.76 .4 & $0.06 \pm 0.01$ & 117 & 1370 & 4480 & $26 \pm 5$ \\
2 & -0.621 & 4.04 .3 & $0.08 \pm 0.01$ & 135 & 650 & 3250 & $22 \pm 3$ \\
3 & -0.829 & 6.57 .5 & $0.57 \pm 0.01$ & 555 & 630 & 3160 & $49 \pm 3$ \\
4 & -0.878 & 11.512 .5 & $0.38 \pm 0.1$ & 947 & 460 & 1960 & $76 \pm 5$ \\
5 & -0.947 & 6.57 .4 & $0.96 \pm 0.03$ & 1524 & $\leqslant 50$ & 830 & $107 \pm 8$ \\
\hline \hline
\end{tabular}

${ }^{a}$ The first value is estimated from the numerical integration procedure used to obtain $\langle\Delta S\rangle$ from magnetization data; the second is the value used to fit Eq. (11) to the experimental values.

${ }^{\mathrm{b}} H^{*}$ is the field corresponding to the minimum of the $\Delta M(H)$ curve, and it approximately corresponds to the saturating field of the parent phase.

${ }^{\mathrm{c}} H_{c}$ is the field that characterizes the response of the magnetization associated with twin boundary motion against the elastic energy [see Eq. (5)], and it is close to the saturation field of the martensitic phase.

applied magnetic field. It first decreases with $H$ for very small fields (this is not observed for sample 5), shows a negative minimum at a given value of the field $\left(H^{*}\right)$, and finally increases and reaches a positive saturation value $\Delta M_{\text {sat }}$ which renders the difference in the saturating magnetic moments of martensite and parent phases.

Recently reported optical observations of the magnetic and structural domain mesostructure formed at the martensitic transition under selected applied fields ${ }^{15,16}$ suggested the actual mechanism responsible for the behavior of the magnetization. These mesostructures are a consequence of the magnetostructural coupling between martensitic variants and magnetic moments, which originates from the strong uniaxial magnetic anisotropy (along the $c$ axis) of the tetragonal martensitic phase. When the martensitic transition takes place at zero field, the nucleation gives rise to martensitic plates formed by parallel strips of twin-related variants. This mesostructure ensures that parent-martensite interfaces satisfy the invariant plane strain condition ${ }^{17}$ which minimizes the elastic strain energy arising from the crystal lattice misfit along the interfacial boundaries. ${ }^{17,18}$ Magnetic domains are formed within each variant in such a way that the magnetization alternates between two opposite values along the corresponding easy axis. When the system is cooled through the transition under a magnetic field larger than the saturating field of the high-temperature phase (which is expected to be close to $\left.H^{*}\right)$, the twin-related variants are magnetized. As the field is increased, due to the strong uniaxial anisotropy of the tetragonal phase, the Zeeman energy difference between neighboring variants is minimized by increasing the fraction of those variants with their easy-magnetization axis forming a smaller angle with the applied field. Finally, transformation under high enough fields results in a magnetically saturated single variant martensitic crystal. It is worth noting that such a mechanism is controlled by the same magnetostructural coupling that accounts for the magnetic shape-memory effect. ${ }^{3,19}$

Within the framework of the preceding scenario, for $H$ $>H^{*}$, the fraction $f$ of variants favored by the application of a magnetic field is determined by the balance between elastic and magnetic energies. ${ }^{3}$ At a given temperature $f$ is obtained as the fraction minimizing the free energy

$$
G(f)=E_{\text {elas }}(f)-\vec{H} \vec{M}(f),
$$

where the elastic energy is given by $E_{\text {elas }}=E_{0} \Phi(f)\left(E_{0}\right.$ is the elastic energy at zero applied field and $\Phi$ is a dimensionless function accounting for the dependence on the transformed fraction). In this expression $\Phi=1$ for $H \leqslant H^{*}$. We will assume, for the sake of simplicity, that the system contains two twin-related variants 1 and 2 . Thus, for $H \leqslant H^{*}$, $f=1 / 2$. For $H \geqslant H^{*}$, the magnetization can be expressed as (strong anisotropy condition) $\vec{M}(f)=\left[f \vec{M}_{1}+(1-f) \vec{M}_{2}\right]$, where $\vec{M}_{1}$ and $\vec{M}_{2}$ are the magnetization of variants 1 and 2 along the corresponding easy axis ( $c$ axis). Let $\theta$ be the angle between $\vec{M}_{1}$ and $\vec{H}$ and $\phi$ the angle between $\vec{M}_{1}$ and $\vec{M}_{2}$, then $\vec{H} \vec{M}(f)=[f \cos \theta+(1-f) \cos (\theta+\phi)] M_{M} H$, where $M_{M}$ is the saturation magnetization of the martensitic phase. Thus, minimization of $G(f)$ with respect to $f$ yields

$$
\frac{d \Phi}{d f}=\left\{\begin{array}{l}
0 \text { for } H \leqslant H^{*}, \\
\frac{H-H^{*}}{H_{c}} \text { for } H \geqslant H^{*},
\end{array}\right.
$$

where the characteristic field $H_{c}$ is

$$
H_{c}=\frac{E_{0}}{M_{M}[\cos \theta-\cos (\theta+\phi)]} .
$$

$H_{c}$ is the field that characterizes the response of the magnetization associated with twin-boundary motion against the elastic energy. Note that the angle $\phi$ for twin-related variants is approximately $\pi / 2$ and, in general, $\theta$ is small. Therefore, $\cos \theta-\cos (\theta+\phi) \simeq 1$. This will be assumed in what follows. The function $\Phi(f)$ is required to be finite for all $f$, such that $(d \Phi / d f)_{f=1 / 2}=0\left(H \leqslant H^{*}\right)$, and that it yields a strong increase of the elastic energy as the favored variant growth with the field $\left[(d \Phi / d f)_{f \rightarrow 1} \rightarrow \infty\right]$. The choice $d \Phi / d f$ $=-\ln [2(1-f)]$ meets these conditions, leading to $f=1$ 
$-\frac{1}{2} e^{-\left(H-H^{*}\right) / H_{c}}$. For $H \leqslant H^{*}$ it is reasonable to assume that $\Delta M$ varies linearly with $H$. Therefore,

$$
\Delta M(H)=\left\{\begin{array}{l}
\Delta M_{\mathrm{sat}}(1-\mu) \frac{H}{H^{*}} \text { for } H \leqslant H^{*}, \\
\Delta M_{\mathrm{sat}}\left[1-\mu e^{-\left(H-H^{*}\right) / H_{c}}\right] \text { for } H \geqslant H^{*},
\end{array}\right.
$$

where $\Delta M_{\text {sat }}$ is the saturation value of $\Delta M$, and $\mu$ $=M_{M} / 2 \Delta M_{\text {sat }}$. $H_{c}$ and $\Delta M_{\text {sat }} / M_{M}$ can be estimated from experimental data by fitting an exponential function to the measured values of $\Delta M$ (in the range $H \geqslant H^{*}$ ). The fits for the different analyzed alloys are plotted in Fig. 3, and the obtained values of the parameters are listed in Table II. The fact that an exponential function yields a good fit to the data (it reproduces the fast saturation of $\Delta M$ ) confirms the suitability of the selected function $\Phi(f)$ in establishing the $f$ dependence of the elastic strain energy. Moreover, it is worth noting that the obtained values of $H_{c}$ clearly decrease with increasing $e / a$. That is, on reducing the temperature range between Curie and martensitic transition temperatures $T_{c}$ $-T_{M}$, which in turn varies linearly with $e / a$.

The contribution arising from the martensitic transition to the magnetization along the direction of the applied magnetic field is taken in the form $M(T, H)=M_{P}(H)+\Delta M(H) F\{[T$ $\left.\left.-T_{M}(H)\right] / \Delta T(H)\right\}$. In this expression, $F\left\{\left[T-T_{M}(H)\right] /\right.$ $\Delta T(H)\}$ is a monotonously decreasing function of width $\Delta T$ such that $F \rightarrow 0$ for $T \gg T_{M}(H)$ and $F \rightarrow 1$ for $T \ll T_{M}(H)$ (for $\Delta T \rightarrow 0, F$ is the Heaviside function). $\Delta T(H)(<0)$ is the temperature range over which the transition spreads when the field changes from $0 \rightarrow H$, and $T_{M}$ an estimation of the martensitic transition temperature. $M_{P}(H)$ is a temperatureindependent quantity. From Eqs. (1) and (2), the averaged field-induced entropy change in the vicinity of the martensitic transition is obtained as

$$
\begin{aligned}
\langle\Delta S(H)\rangle= & \frac{1}{\Delta T(H)} \int_{0}^{H^{*}} \Delta M\left(H \leqslant H^{*}\right) d H \\
& +\frac{1}{\Delta T(H)} \int_{H^{*}}^{H} \Delta M\left(H \geqslant H^{*}\right) d H
\end{aligned}
$$

where we have taken into account the fact that

$$
\int_{\Delta T} \frac{\partial F}{\partial T} d T=1
$$

In the preceding expression $\Delta T(H)$ is given by

$$
\Delta T(H) \simeq \Delta T_{t}(H)+\frac{d T_{M}}{d H} H,
$$

where $\Delta T_{t}(H)$ is the temperature range over which the transition spreads for a given field $H$ and $\left(d T_{M} / d H\right) H$ is a measure of the shift of the transition temperature induced by the field. From the magnetization measurements it is obtained that for moderate fields $\Delta T_{t}$ is almost independent of $H .^{20}$ Moreover, since $d T_{M} / d H$ is small in Ni-Mn-Ga alloys (see
Table II), for fields that are not too high it is reasonable to assume that $\Delta T$ is independent of $H$.

(i) For $H \leqslant H^{*}$, from the preceding Eqs. (6) and (7), a quadratic dependence of $\langle\Delta S(H)\rangle$ on the field is obtained in this range of fields

$$
\langle\Delta S(H)\rangle=(1-\mu) \frac{H^{*} \Delta M_{\mathrm{sat}}}{2 \Delta T}\left(\frac{H}{H^{*}}\right)^{2} .
$$

(ii) For fields $H \geqslant H^{*}$, but which are not high enough to induce the whole transition, that is, $H$ $<\left|\Delta T_{t}\right|\left(d T_{M} / d H\right)^{-1}$, the averaged field induced entropy change turns out to be

$$
\begin{aligned}
\langle\Delta S(H)\rangle= & -(1+\mu) \frac{\Delta M_{\mathrm{sat}}}{2 \Delta T} H^{*}-\mu \frac{H_{c} \Delta M_{\mathrm{sat}}}{\Delta T} \\
& \times\left(1-e^{-\left(H-H^{*}\right) / H_{c}}\right)+\frac{\Delta M_{\mathrm{sat}}}{\Delta T} H .
\end{aligned}
$$

Actually, this is a suitable approximation in order to analyze the behavior of the field-induced entropy change in $\mathrm{Ni}$ $\mathrm{Mn}-\mathrm{Ga}$ in a broad range of fields. ${ }^{21}$ By using the quantities $H^{*}, H_{c}, \mu$, and $\Delta M_{\text {sat }}$ given in Table II, it is possible to fit Eq. (11) to the experimental data shown in Fig. 2. In such a fit, $\Delta T$ is the only free parameter, which permits the adjustment of the vertical scale. The fitted values of $\Delta T$ are also given in Table II, where they can be compared with the corresponding values estimated from the integration procedure used to calculate $\langle\Delta S(H)\rangle$. The agreement between model and experiments is very satisfactory for all studied alloys (the maximum discrepancy between the two estimated values of $\Delta T$ is less than $15 \%$ ).

The last term in Eq. (11) can be expressed in the following form:

$$
\frac{\Delta M_{\mathrm{sat}}}{\Delta T} H=-\frac{\Delta T_{M}}{\Delta T_{t}+\Delta T_{M}} \Delta S_{t},
$$

where $\Delta T_{M}=T_{M}(H)-T_{M}(0)$ is the shift in the transition temperature due to the magnetic field and $\Delta S_{t}$ is the whole entropy change taking place at the martensitic transition which is known to be independent of $H^{12}$ Equation (12) is obtained taking into account the Clausius-Clapeyron equation $H=-\left(\Delta S_{t} / \Delta M_{\text {sat }}\right)\left[T_{M}(H)-T_{M}(0)\right]$. For small $H$, $\Delta T_{M} \ll \Delta T_{t} \quad$ so that $\Delta T_{M} /\left(\Delta T_{t}+\Delta T_{M}\right) \simeq \Delta T_{M} / \Delta T_{t} \simeq \alpha$, where $\alpha$ is the transformed fraction of martensite induced by the application of the magnetic field.

(iii) For large enough fields such that $H$ $>\left|\Delta T_{t}\right|\left(d T_{M} / d H\right)^{-1}>H_{c}\left(>H^{*}\right)$ the whole transition is induced $(\alpha \rightarrow 1)$, and the first two terms in Eq. (11) have reached their saturation values $(f \rightarrow 1)$, therefore,

$$
\begin{aligned}
\langle\Delta S(H)\rangle \simeq & -\frac{\Delta M_{\mathrm{sat}}}{\Delta T}\left[\frac{1}{2}(1+\mu) H^{*}+\mu H_{c}\right] \\
& -\frac{\Delta T_{M}}{\Delta T_{t}+\Delta T_{M}} \Delta S_{t} .
\end{aligned}
$$


TABLE III. Contributions to the magnetocaloric effect as given by Eq. (11), evaluated at $H=H_{c}$ for all the studied alloys. All data are given in $\mathrm{J} / \mathrm{K}$ mol.

\begin{tabular}{cccc}
\hline \hline Alloy No. & First term & Second term & Third term \\
\hline 1 & 0.0239 & 0.0738 & -0.0082 \\
2 & 0.0139 & 0.0718 & -0.0102 \\
3 & 0.0064 & 0.0227 & -0.0233 \\
4 & 0.0063 & 0.0210 & -0.0150 \\
5 & 0.0006 & 0.0111 & -0.0172 \\
\hline \hline
\end{tabular}

Notice that in some cases the term $\left(\Delta M_{\text {sat }} / \Delta T\right)[(1$ $\left.+\mu) H^{*} / 2+\mu H_{c}\right]$ can be very small compared to $\Delta S_{t}$. For instance, the ratio between these two quantities is $\sim 5 \%$ for alloys 3 and 4, and less than $2 \%$ for alloy 5 . In the limit of very high fields, $\Delta T_{t} \ll \Delta T_{M}$, then $\langle\Delta S(H)\rangle \rightarrow \Delta S_{t}$. The transition entropy change thus represents the maximum (in absolute value) reachable value of the averaged field induced entropy change.

It is now interesting to analyze separately the three contributions to $\langle\Delta S(H)\rangle$ in Eq. (11). The first term on the righthand side of this equation is related to the initial decrease of $\Delta M(H)$. This term is proportional to $H^{*}$, which is usually a weak field compared with $H_{c}$ (see Table II), and therefore this term leads to a small contribution to the magnetocaloric effect. The second term provides the contribution which arises from the magnetostructural coupling between martensitic variants and magnetic moments. This contribution is positive and is dominant for magnetic fields less than $H_{c}$ (see Fig. 2). Interestingly, its magnitude is proportional to the saturation magnetization of the martensitic phase $M_{M}$ $=2 \mu \Delta M_{\text {sat }}$, and to $H_{c}$. From our analysis both $H_{c}$ and $M_{M}$ (Ref. 22) are maximum close to the stoichiometric $\mathrm{Ni}_{2} \mathrm{MnGa}$ composition $(e / a=7.5)$. This dependence of the magnetization saturation on $e / a$ is in agreement with recently reported data. $^{23,24}$ This explains that the maximum positive contribution to the field-induced entropy change is obtained for alloys 1 and 2 with $e / a$ closer to 7.5.

The third contribution to $\Delta S_{\max }$ is negative and is the expected contribution to the magnetocaloric effect in the vicinity of a magnetostructural transition. ${ }^{25}$ Figure 2 shows that this contribution to $\langle\Delta S(H)\rangle$ depends linearly on the applied field. Moreover, the relative importance of this term increases with increasing $\Delta T_{M}$ which, for a given field, depends on $d T_{M} / d H$. This derivative can be obtained from the Clausius-Clapeyron equation as the ratio between $\Delta M_{\text {sat }}$ and $\Delta S_{t}$. Notice that this derivative provides an estimation of the strength of the microscopic spin-phonon coupling in the system. In our case we see that it strongly increases with $e / a$ (see Table II).

A quantitative comparison of the three contributions to the magnetocaloric effect for all the studied samples can be performed by evaluating them at $H=H_{c}$. Results are listed in Table III.

\section{SUMMARY AND CONCLUDING REMARKS}

From a general viewpoint, the magnetocaloric effect is an interesting property in magnetic materials that depends sen- sitively on the complex magnetic structures that develop at different length scales during magnetization processes. The magnetocaloric effect is defined as the adiabatic temperature change arising from the application/removal of a magnetic field $H$. A positive magnetocaloric effect corresponds to an adiabatic increase (decrease) of temperature when $H$ is applied (removed), and it is associated to a negative (positive) entropy change on isothermal application (removal) of magnetic field.

In the vicinity of the martensitic transition of Ni-Mn-Ga, the magnetocaloric effect is controlled by the interplay between magnetic and structural degrees of freedom. The phenomenon occurs simultaneously at multiple length scales from the microscopic atomic scale to mesoscopic scales, which range from the scale of magnetic domains inside martensitic variants to the scale of the twin variants. In the present work, we have evaluated the contribution from these different scales to the magnetocaloric effect.

We have seen that the dependence of the magnetic properties of Ni-Mn-Ga on e/a leads to a magnetocaloric effect which also shows a strong $e / a$ dependence. Two characteristic magnetic fields are relevant in order to evaluate the relative importance of each contribution to the magnetocaloric effect, namely $H^{*}$ and $H_{c}$, which approximately correspond to the saturating fields in the parent and martensitic phases, respectively. We have also found that both $H^{*}$ and

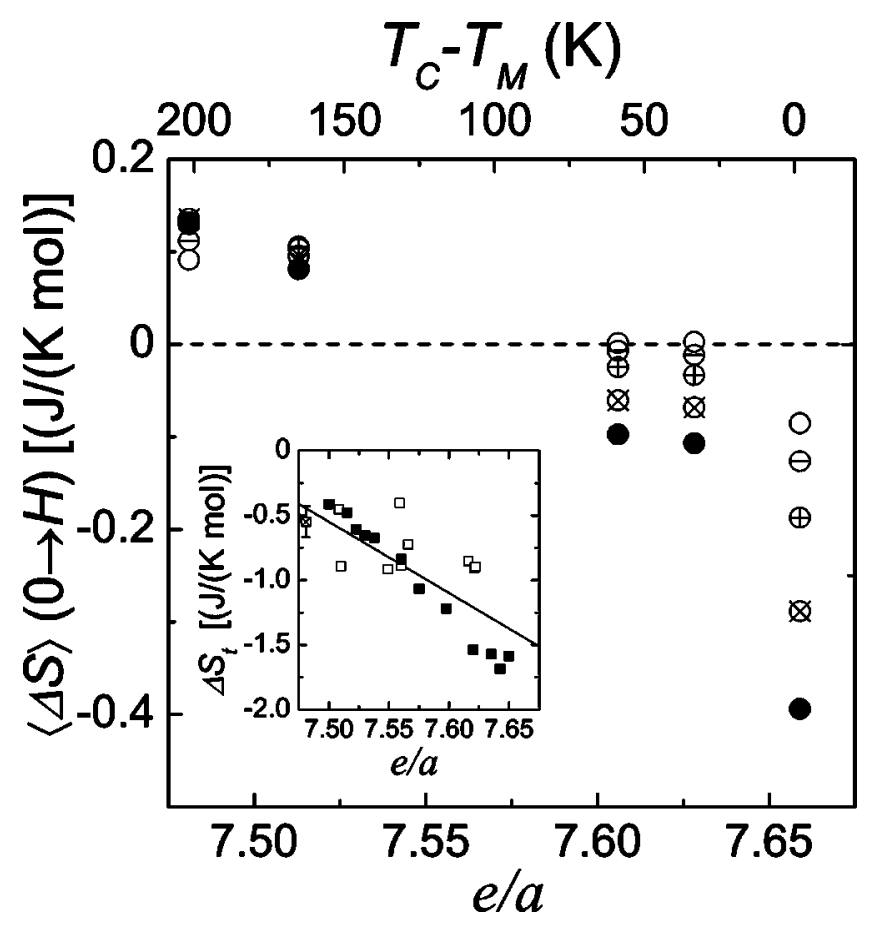

FIG. 4. Average field-induced entropy change $\langle\Delta S\rangle$ as a function of $e / a$ and $T_{c}-T_{M}$ at selected values of the inducing field $H$ $=5 \mathrm{kOe}(\bigcirc), 7 \mathrm{kOe}(\ominus), 10 \mathrm{kOe}(\oplus), 15 \mathrm{kOe}(\otimes)$, and $20 \mathrm{kOe}$ (๑). Inset: Transition entropy change $\Delta S_{t}$ as a function of $e / a(\nabla$ from Ref. 12, $\square$ from Ref. $26\left[\Delta S_{t}\right.$ is estimated as the ratio between the latent heat and $T_{0}=\left(M_{s}+A_{f}\right) / 2$ with $M_{s}$ and $A_{f}$ the temperatures of the starting and finishing temperatures of the forward (cooling) and reverse (heating) transitions], and $\mathbf{\square}$ from Ref. 27). The continuous line is a linear fit to these data. 
$H_{c}$ decrease as $e / a$ increases, that is, as $T_{c}-T_{M}$ goes to zero (see Tables I and II).

For fields in the range between $H^{*}$ and $H_{c}$ (low fields), magnetization occurs throughout a twin reorientation process in the transition region due to the high anisotropy of the martensitic phase (tetragonal symmetry). This mechanism mainly controls the magnetocaloric effect in such a range of fields, and it gives $\Delta S>0$, which means that the sample increases its temperature by adiabatic demagnetization. The maximum value for this contribution is achieved for alloys with a composition close to the Heusler composition (which transforms below room temperature). In contrast, for high fields $\left(H>H_{c}\right)$ the microscopic coupling, responsible for the change of intrinsic magnetic properties at the phase transition, is at the origin of the magnetocaloric effect. This effect is adequately accounted for by the derivative $d T_{M} / d H$ which gives the rate of change of the martensitic transition temperature with an applied magnetic field and can be obtained from the Clausius-Clapeyron equation. This contribution yields $\Delta S<0$, and is maximum in absolute value for alloys with the martensitic and Curie transition temperatures (above room temperature) close to each other. In this case, the magnetocaloric properties are comparable to those reported for $\mathrm{Gd}_{5}\left(\mathrm{Si}_{x} \mathrm{Ge}_{1-x}\right)_{4}$ (Ref. 9) or MnAs-based compounds, ${ }^{10,11}$ which display the giant magnetocaloric effect in the vicinity of a magnetostructural transition, at which the crystallographic change is accompanied by a discontinuity of magnetic order which changes from paramagnetic to ferromagnetic. The maximum values of the field-induced en- tropy in the Ni-Mn-Ga alloy with $T_{M} \sim T_{c}$ are approximately $-30 \mathrm{~J} / \mathrm{K} \mathrm{kg}$ for a field of $15 \mathrm{kOe},{ }^{14}$ which is even larger than maximum values reported for $\mathrm{Gd}_{5}\left(\mathrm{Si}_{x} \mathrm{Ge}_{1-x}\right)_{4}$ and MnAsbased materials, which are of the order of $-15 \mathrm{~J} / \mathrm{K} \mathrm{kg}$ for a field of $20 \mathrm{kOe}$.

Figure 4 summarizes the obtained dependence on $e / a$ of the magnetocaloric effect. The figure shows the averaged field-induced entropy change at selected values of the applied field, as a function of $e / a$ and of $T_{c}-T_{M}$. These values are compared with the values of the transition entropy change which provide, for each value of $e / a$, the limiting (high fields) value of $\langle\Delta S(H)\rangle . \Delta S_{t}$ as a function of $e / a$ is shown in the inset of Fig. 4. The values are taken from Refs. $12,26,27$. The values of $\Delta S_{t}$ given in Table II correspond to interpolated values obtained from a least square fit (continuous line in the figure) to experimental data. Notice that in all cases the field-induced entropy change is far from the value of the transition entropy change which represents the maximum absolute reachable value. This means that the magnetic field is not high enough to enable the transformation of the whole sample.

\section{ACKNOWLEDGMENTS}

This work has received financial support from CICyT (Projects No. MAT2001-3251, MAT2000-0858) and DURSI (Project No. 2001SGR00066). J.M. and F.C. acknowledge financial support from DURSI.
${ }^{1}$ L1. Mañosa and A. Planes, Adv. Solid State Phys. 40, 361 (2000), and references therein.

${ }^{2}$ Shape Memory Materials, edited by K. Otsuka and C.M. Wayman (Cambridge University Press, Cambridge, 1998).

${ }^{3}$ R.C. O’Handley, J. Appl. Phys. 83, 3263 (1998).

${ }^{4}$ R.D. James and M. Wutting, Philos. Mag. A 77, 1273 (1998).

${ }^{5}$ A.N. Lavrov, S. Komiya, and Y. Ando, Nature (London) 418, 385 (2002).

${ }^{6}$ F. Hu, B. Shen, and J. Sun, Appl. Phys. Lett. 76, 3460 (2000).

${ }^{7}$ F. Hu, B. Shen, J. Sun, and G. Wu, Phys. Rev. B 64, 132412 (2001).

${ }^{8}$ A.M. Tishin, in Handbook of Magnetic Materials, edited by K.H.J. Buschow (North-Holland, Amsterdam, 1999), Vol. 12, Chap. 4, pp. 395-524.

${ }^{9}$ V.K. Pecharsky and K.A. Gschneidner, Jr., Phys. Rev. Lett. 78, 4494 (1997).

${ }^{10}$ H. Wada and Y. Tanabe, Appl. Phys. Lett. 79, 3302 (2001).

${ }^{11}$ O. Tegus, E. Brück, K.H.J. Buschow, and F.R. de Boer, Nature (London) 415, 450 (2002).

${ }^{12}$ J. Marcos, A. Planes, Ll. Mañosa, F. Casanova, X. Batlle, A. Labarta, and B. Martínez, Phys. Rev. B 66, 224413 (2002).

${ }^{13}$ The compositional dependence of $T_{M}$ can be conveniently reduced in terms of $e / a$. Usually it is assumed that the number of valence electrons per atom for $\mathrm{Ni}, \mathrm{Mn}$, and $\mathrm{Ga}$ are 10 $\left(3 d^{9.9} 4 s^{0.1}\right), 7\left(3 d^{5} 4 s^{2}\right)$, and $3\left(4 s^{2} 4 p^{1}\right)$, respectively. See, V.A. Chernenko, Scr. Metall. 40, 523 (1999).

${ }^{14}$ L. Paretti, M. Solzi, F. Albertini, and A. Paoluzi, Eur. Phys. J. B
32, 303 (2003).

${ }^{15}$ Q. Pan and R.D. James, J. Appl. Phys. 87, 4702 (2000).

${ }^{16}$ H.D. Chopra, C. Ji, and V.V. Kokorin, Phys. Rev. B 61, R14 913 (2000).

${ }^{17}$ A.G. Khachaturyan and G.A. Shatalov, Sov. Phys. JETP 29, 557 (1969); A.G. Khachaturyan, S.M. Shapiro, and S. Semenovskaya, Phys. Rev. B 43, 10832 (1991).

${ }^{18}$ G.R. Barsch, B. Horovitz, and J.A. Krumhansl, Phys. Rev. Lett. 59, 1251 (1987).

${ }^{19}$ S.-Y. Chu, A. Cramb, M. De Graef, D. Laughlin, and M.E. McHenry, J. Appl. Phys. 87, 5777 (2000).

${ }^{20}$ The analysis of experimental data reveals that $\Delta T_{t}$ shows small changes for $H<H^{*}$.

${ }^{21}$ Taking $\left|\Delta T_{t}\right| \simeq 10 \mathrm{~K}$ and $d T / d H \simeq 50 \mathrm{mK} / \mathrm{kOe}$ (see Table II), we find that Eq. (11) is valid for $H<200 \mathrm{kOe}$.

${ }^{22}$ See Table II where an estimation of the saturation magnetization of the martensitic phase can be obtained from $1 / \mu$ and $\Delta M_{\text {sat }}$.

${ }^{23}$ K. Tsuchiya, A. Ohashi, D. Ohtoyo, H. Nakayama, M. Umemoto, and P.G. McCormick, Mater. Trans., JIM 41, 938 (2000).

${ }^{24}$ X. Jin, M. Marioni, D. Bono, S.M. Allen, R.C. O'Handley, and T.Y. Hsu, J. Appl. Phys. 91, 8222 (2002).

${ }^{25}$ F. Casanova, X. Batlle, A. Labarta, J. Marcos, L1. Mañosa, and A. Planes, Phys. Rev. B 66, 100401(R) (2002).

${ }^{26}$ V.A. Chernenko, E. Cesari, V.V. Kokorin, and I.N. Vitenko, Scr. Metall. Mater. 33, 1239 (1995).

${ }^{27}$ V.V. Khovailo, K. Oikawa, T. Abe, and T. Takagi, J. Appl. Phys. 93, 8483 (2003). 